KENTRON

EVUE PLURIDISCIPUINAIRE

DU MONDE ANTIQUE

\section{Kentron}

Revue pluridisciplinaire du monde antique

$30 \mid 2014$

L'emprunt, une pratique d'écriture

\title{
Emendativncvlae Malaterrenae IV
}

\section{Olivier Desbordes}

\section{OpenEdition}

Journals

Édition électronique

URL : http://journals.openedition.org/kentron/547

DOI : $10.4000 /$ kentron.547

ISSN : 2264-1459

\section{Éditeur}

Presses universitaires de Caen

\section{Édition imprimée}

Date de publication : 1 octobre 2014

Pagination : 147-158

ISBN : 978-2-84133-506-0

ISSN : 0765-0590

Référence électronique

Olivier Desbordes, «Emendativncvlae Malaterrenae IV », Kentron [En ligne], 30 | 2014, mis en ligne le 19 décembre 2016, consulté le 18 novembre 2020. URL : http://journals.openedition.org/kentron/547 ; DOI : https://doi.org/10.4000/kentron.547

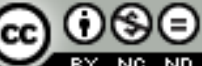

Kentron is licensed under a Creative Commons Attribution-NonCommercial-NoDerivatives 3.0 International License. 


\section{EMENDATIVNCVLAE MALATERRENAE IV}

Si dans l'étude et l'émendation du texte de Geoffroi Malaterra, la critique peut, en règle générale, faire fond sur quatre manuscrits ${ }^{1}$, elle doit néanmoins, en plus d'un endroit, limiter sa visée au seul manuscrit $Z^{2}$. Dans l'édition qu'E. Pontieri a donnée de cette chronique ${ }^{3}$, les leçons de $\mathrm{Z}$ ne sont appréhendées qu'indirectement, par le

1. Ces mss se divisent en deux familles. Les plus importants pour la constitution du texte, A (Palermo, Bibl. Centr. della Regione Siciliana, X.A.16) et C (Catania, Bibl. Univ., Vent. Arm. 1 99), sont dans la première, $\alpha$; la seconde, $\beta$, issue d'une copie altérée de l'original, présente des essais de correction et autres remaniements déterminés par la corruption du modèle. Elle se subdivise elle-même en deux groupes bien distincts: le premier est formé du ms. Z (Barcelona, Bibl. de Catalunya y Central, 0996), dont dérive directement l'édition princeps (= P), parue à Saragosse en 1578; à l'autre appartiennent le ms. B (Palermo, Bibl. della Soc. Sicil. di Storia Patria, I B 28) et ses copies, directe, Be (Besançon, Bibl. mun., 675), et subséquente, D (Palermo, Bibl. Comm., Qq.E.165). Sur les principes qui doivent servir de points de départ dans l'établissement du texte de Malaterra, voir Resta 1964, résumé en dernier lieu par Lucas-Avenel 2008, 32-38.

2. Seul $\mathrm{Z}$ possède le texte intégral de la chronique de Malaterra - compte non tenu des pertes subies par cet état du texte au cours de sa transmission. Sans vouloir en dresser une liste complète, nous signalerons ici à titre d'exemples celles dont la nature ou l'étendue constitue un obstacle réel à l'intelligence de la phrase mutilée: I $23^{*}$ (p. 20,6-8) a fratre [degenerem - a fratre] recedens; I $31^{*}$ (p. 22,19 sq.) fratri [puellae - frater] suus; II praef. (p. 29,3) facientes [ad easdem - unaquaeque res] describenda; $\mathrm{II} 7^{\star}$ (p. 31,33) caelorum [istis - caelorum] non esse; II 26 (p. 38,21) mei [ecce - meum mihi] inimicari; II 28 (p. 39,20) sibi [accersiens - sibi] conuentionem; II 29 (p. 39,33 sq.) iuuenculam [timidam et in quantum] audebat; II 44 (p. 52,16) accidisse [cuius - fuisse] excusationibus; III 10 (p. 61,29 sq.) erat [cum filia - dederat] totam Siciliam; III 22 (p. 70,14-17) concessum [et ab utrisque - concessum] renuntiant; IV $3^{*}$ (p. 87,5 sq.) pecunia [classem - nulla] classe; IV 22 (p. 100,33 sq.) peruaserat [retinere - quae peruaserat] duci restituit; IV 29 (p. 107,11) Siciliam [et sui-habitam] uel habendam. Ces lacunes résultent en règle générale d'un passage « du même au même»; quatre de ces accidents - ceux que distingue l'astérisque - remontent au plus proche commun ancêtre des mss $Z$ et $B$. Il reste qu'en plus d'un endroit, des éléments de phrase ont disparu sans que le sens en paraisse altéré: cf. I 3 (p. 8,21) uenerant [Normanni dicti] terram; I 7 (p. 11,11 sq.) peruenerunt [cuius ciues - congrediuntur] Archadius; II 29 (p. 40,14) taliter impug[natio - inimicos impug]nando; II 33 (p. 42,27) nec illi uersus istos [nec isti uersus illos] transire.

3. Cf. Pontieri 1927-1928. Les indications de page, suivies d'un chiffre pour la ligne, données entre parenthèses renvoient à cette édition. Les textes que nous citons ont tous été contrôlés sur les mss. 
truchement de l'édition princeps. Et quand les manuscrits qu'il a réunis ${ }^{4}$ sont tous en défaut, le savant italien n'a d'autre ressource que de reproduire le premier en date des textes imprimés. C’est le cas pour la seconde épître dédicatoire, que deux seulement des quatre témoins ont conservée ${ }^{5}, \mathrm{~A}$ - partiellement - et Z: à po[etria (p. 4,15) s'ouvre dans A une lacune qui, s'étendant jusqu'au début du livre I (maxima] classe [p. 7,8$]$ ), a emporté la fin de la lettre. Dans l'édition Pontieri, le texte de cette séquence reflète exactement - exception faite pour deux fautes d'impression ${ }^{6}$ - celui que G. Zurita avait établi dans les marges de $\mathrm{Z}$ et soumis aux presses de Domenico de Portonaris ${ }^{7}$.

4. L'édition qu'a donnée E. Pontieri dans le «nouveau Muratori» fait connaître A et B, que G.B. Caruso avait déjà mis à profit dans sa révision du texte de la princeps, mais aussi $\mathrm{C}$ et $\mathrm{D}$. Si cette édition est réputée déficiente sur le plan philologique et très contestable - "assolutamente inadeguata", suivant le jugement de D'Angelo 2003, 216 -, bien qu'elle trouve encore un bon accueil auprès des historiens (cf. Toubert 2005, 351 sq.), c'est à cause moins d'une appréciation inexacte de la valeur intrinsèque des sources - manuscrites et imprimée - et des relations qu'elles entretiennent entre elles que d'une connaissance défectueuse des lois de la critique et des devoirs de l'éditeur (cf. Resta 1964, 32). Sans doute $\mathrm{Z}$ a-t-il été indûment rapproché de $\mathrm{A}$ et de $\mathrm{C}$, au nom d'une illusoire communauté d'origine (cf. Pontieri 1927-1928, Prefazione, p. LIII, LV et LVI), mais la valeur propre de ces deux derniers témoins et leur intérêt pour la constitution du texte (ibid., p. LVI sq.; cf. aussi Resta 1964, 24; 30), le rôle de supplétif que tient $C$ vis-à-vis de A ont été reconnus et sont affirmés avec force dans les conclusions énoncées p. LVI. Il reste que la promotion de B, élevé par défaut - du fait de l'élimination de A consécutive à son état fragmentaire - au rang de ms. le plus ancien de Malaterra, et la conviction que la piètre qualification professionnelle du scribe qui a transcrit ce codex est la meilleure garantie contre toute forme d'interpolation et, partant, de l'excellence de son témoignage (ibid., p. LV) ont amené Pontieri à accorder, aux dépens de $\mathrm{C}$, une prédilection parfois aveugle, souvent injuste, à cet état de la tradition. Enfin - et c'est le grief que nourrit la lecture de presque chaque page de l'édition -, l'exécution matérielle laisse beaucoup à désirer sous le rapport de l'exactitude: les collations sont incomplètes, les erreurs de lecture et d'attribution fréquentes. Maintes leçons précieuses sont ainsi passées sous silence.

5. B et $\mathrm{D}$ ont perdu les pièces liminaires et le sommaire du livre I. C ne commence qu'au chapitre 2 du même livre I; les premiers mots conservés sont se agere posse cognosceret (p. 7,16). Mutilé au commencement, ce témoin est également incomplet de la fin: s'arrêtant en IV 22 sur les mots quod ut faceret primo (p. 101,3), il restreint alors le champ d'action de la critique aux seuls représentants de la famille $\beta$.

6. faciat (p. 4,37, pour faciant) se heurte à une impossibilité grammaticale, l'accord des verbes de la phrase étant déterminé par un pluriel, alii. Quant à potata (p. 4,41, pour putata), que tous les traducteurs modernes ont singulièrement pris au sérieux - malgré, entre autres, Virgile, georg. 2, 407, ou la Vulgate, Lv 25,3 sq., et Is 5,6 -, il semble que ce soit, non une retouche volontaire - justifiée à contresens par des considérations techniques: l'arrosage, ou l'irrigation, plutôt que la taille -, mais une faute de compositeur, entrée déjà auparavant par inadvertance dans Muratori 1724, 547. Sauf le hasard d'une rencontre, cette innovation devait être consignée dans l'apparat critique. On relève la même métaphore agricole dans ce passage de Pierre Lombard, Comm. in Ps. 79,16, appliquée cette fois à la maison d'Israël: [...] uinea est gens Iudaeorum [...]; quia inter turbas umbratiles peccantium fruge sanctorum ornatur, sicut uinea fructu ornatur inter folia, et fossa tribulationibus et martyrio putata plus crescit sicut uinea [...].

7. Voici la liste des altérations de $\mathrm{Z}$ redressées par $\mathrm{G}$. Zurita $\left(=\mathrm{Z}^{2}\right)$ dans ce morceau de texte où $\mathrm{A}$ ne compte plus. Sont marquées d'un astérisque les interventions critiques attribuables à une seconde 
Or, G. Resta a démontré par des preuves décisives que ce manuscrit $\mathrm{Z}$ n'est pas suffisant pour la constitution du texte définitif de Malaterra ${ }^{8}$. Il y a donc encore des lectures fautives - à tout le moins douteuses - passées inaperçues dans les parties de la chronique pour lesquelles nous devons, toute forme de contrôle extérieur étant impossible, nous accommoder du témoignage de $\mathrm{Z}$.

L'examen de la portion de lettre qui commence à l'endroit où le manuscrit $\mathrm{A}$ est défaillant aura livré au moins deux leçons de $\mathrm{Z}$ qui, nous en sommes convaincu, ne sont que des altérations de la rédaction primitive.

La première de ces expressions dont la forme insolite justifie la défiance est contenue dans ce membre de phrase: (p. 4,21 sq.) [...] me uero, quasi uacantem et nulli exercitio deditum, quadam manu pulsationis uigilantiorem reddere satagit. L'association de mots quadam manu pulsationis donne une tournure étrange ${ }^{9}$ : l'emploi métaphorique de manus avec pour complément le génitif d'un substantif abstrait n'est pas en cause ${ }^{10}$; mais le sens qu'on peut dégager ici de cette construction dans le respect de la syntaxe - «d' [une] main qui lui sert à frapper» - amène à douter de l'authenticité de cette lecture de Z. D'autre part, l'indéfini déterminant manu est inapproprié. Ces anomalies s'évanouissent ensemble, si l'on sait reconnaître dans

main sans qu'on puisse en déterminer l'origine: talis $Z^{2}$ : tali $Z^{1}$ (p. 4,17); ieiunum $Z^{2}$ : ieiunii $Z^{1}$ (p. 4,17); oportuit $\mathrm{Z}^{2}$ : poterit $\mathrm{Z}^{1}$ (p. 4,18); inimico $\mathrm{Z}^{2}$ : inimicis $\mathrm{Z}^{1}$ (p. 4,26); utcunque $\mathrm{Z}^{2}$ : utrumque $\mathrm{Z}^{1}$ (p. 4,27); attigerint $\mathrm{Z}^{*}$ : attingerint $\mathrm{Z}^{1}$ (p. 4,27); detrahendo $\mathrm{Z}^{2}$ : detrahendi $\mathrm{Z}^{1}$ (p. 4,30); laude $\mathrm{Z}^{*}$ : laudem $Z^{1}$ (p. 4,30); figentes $Z^{2}$ : fingentes $Z^{1}$ (p. 4,34); ornate $Z^{2}$ : ornato $Z^{1}$ (p. 4,35); cultu $Z^{2}$ : cultus $Z^{1}$ (p. 4,41). En cinq occasions, le texte imprimé diffère de $Z$ : tentantium] conantium $Z$ (p. 4,26); sin] si Z (p. 4,29); et] etiam Z (p. 4,33); ut add. ed. pr. (p. 4, 33); tantum post seipsos om. ed. pr. (p. 4,36). Au début du livre I, dans le passage pour lequel A n'existe pas, $P$ entre en concurrence avec $\mathrm{B}$ et $\mathrm{D}$, et le texte constitué par Pontieri présente, contre toute méthode, un mélange de lectures de provenances diverses. Une préférence injustifiée a été accordée à certaines leçons de B, que la construction ou le sens n'imposent pas sans réserves: ainsi et sic uocabatur (p. 7,4) a supplanté nuncupabatur (Z, P); parta audacia (p. 7,5), pirata a dacia (Z, P); ubi (p. 7,7), quo (Z, P). Enfin, la collation n'est pas sans faute: lire Norueya (et non Norveja, p. 7,5); plurium (au lieu de plurimum, p. 7,5 [apparat]); usque (p. 7,6), possible mélecture d'une forme abrégée, est attribué tacitement à $\mathrm{B}$, qui porte en fait uersus, comme $\mathrm{P}$ et $\mathrm{D}$.

8. Voir Resta 1964, 12-14. Confrontant au texte de la première lettre de dédicace tel que Pontieri l'a établi l'état de la tradition manuscrite recueilli dans A, il a su reconnaître dans ce témoin un certain nombre de leçons authentiques (cf. Resta 1964, 48). Il aurait pu du reste rétablir d'autres variantes, d'autant plus dignes de retenir l'attention qu'elles concordent avec les lectures du second témoin qui a transmis les épîtres dédicatoires: voir Lucas-Avenel 2008, 41.

9. Cette singularité d'expression n'apparaît pas dans les versions modernes de Malaterra, les traducteurs ne s'attachant pas à rendre précisément le latin de Pontieri.

10. La même épître offre plus bas une expression ressemblante: (p. 4,22-25) Rogo itaque uos ut [...] pedem uestri fauoris porrigatis. 
l'expression réputée corrompue un spécimen de "metathesis of inflexion ${ }^{11}$, faute de copie consistant à altérer deux mots - souvent contigus, mais pas nécessairement en intervertissant leur désinence: ici, il faut écrire quadam manus pulsatione - «en m'assenant comme une bourrade avec son poing». Et nous pouvons alléguer au moins deux passages en tout point comparables qui prouvent sans contredit que le copiste de Z - ou l'un de ceux qui l'ont précédé dans ce rameau de la tradition était susceptible de lapsus de cette nature: cf. p. 4,5 sq. [...] ipsos a quibus facta sunt quadam uita (A: uitae Z, edd.) memoriae (A: -ria Z, edd.) ${ }^{12}$; I 8 (p. 12,12 sq.) [...] Calabriam et quaecumque Graecorum iuris esse sciebant uastantes (C B : uastant Z, ed. pr.) percurrunt (C B: -rentes Z, ed. pr.) ${ }^{13}$.

La phrase suivante, (p. 4,39-42) Ego uero quaecumque dictauero uobis corrigenda et rosis uestrae scientiae exornanda reputabo, ut uinea, a uobis exarata, cultu uestrae scientiae putata, uberiores fructus reddens, in maiorem laudem et gratiam perueniat principis, présente elle aussi une difficulté: reputabo n'y donne pas un sens satisfaisant, sauf à lui attribuer une acception particulière, que l'usage général de ce verbe ne semble pas autoriser ${ }^{14}$. Or, les circonstances se trouvent être favorables: même si nous n'avons pas ici le témoignage de A pour contrôler celui de $Z$, nous ne sommes pas réduit à chercher une correction par la seule conjecture. En effet, la première lettre de dédicace renferme une expression exactement comparable, dans laquelle $\mathrm{Z}$ porte reputari, que tous les éditeurs ont adopté d'une façon mécanique jusqu'à ce que Resta ${ }^{15}$ impose la leçon mieux autorisée de A, praesentari, que le sens

11. Cette dénomination est due à A.E. Housman: cf. Diggle \& Goodyear 1972, 378. Elle est illustrée, entre autres, par Stace, silu. 3,1,18 angusto bis seni (devenu angusti bis seno dans nos mss) et Ovide, am. 2,5,27 qualia credibile est non Phoebo (Bentley: -bum codd.) ferre Dianam (Bentley: -nae codd.).

12. Ces lectures de A concordent avec Cicéron, de orat. 2, 36 - preuve supplémentaire, s'il en était besoin, que les leçons de ce témoin doivent, a priori, jouir d'une grande autorité.

13. De ces deux endroits, seul le second est signalé dans l'apparat de Pontieri - et encore, incorrectement: cf. p. 12,13 [apparat] uastantes, percurrentes P. Le premier en a été purement et simplement exclu, bien que cette leçon dédaignée de A mérite seule d'être prise en considération.

14. Les traducteurs de Pontieri ne s'accordent pas sur ce qu'il faut entendre. Loud 2005, 3, donne à reputabo la valeur du verbe simple: «I shall think»; Lucas-Avenel 2001, 6 («je considère»), et Lo Curto 2002, 25 ("spero»), ne rendent pas le futur; quant à Wolf 2005, 44 («I submit for you to correct»), il attribue au verbe la signification que le contexte semble demander, sans lui non plus respecter le temps. Aucune subtilité d'interprétation n'éloigne la suspicion d'impropriété qui pèse sur cette leçon - n'eût-elle paru manifestement corrompue à personne auparavant.

15. Cf. Resta 1964, 24 et 48. L'embarras des traducteurs devant le texte de Pontieri est ici de nouveau perceptible: Loud 2005, 2, escamote la difficulté ("I therefore entreat that [...] my book may be well received»); Lo Curto 2002, 21, se fiant au contexte, anticipe en quelque sorte la correction ( Io quindi mi auguro che [...] questo libro possa autorevolmente presentarsi gradito al principe Ruggero »), pour oublier ensuite l'incidence de cet infléchissement du sens; enfin, Wolf 2005, 41, se 
réclame $^{16}:(\mathrm{p} .3,9)[\ldots]$ librum hunc praesentari (A : reputari Z) expostulo. Nous pouvons induire de ce rapprochement que le même mot, lu dans le même modèle en deux endroits différents, aura occasionné dans $\mathrm{Z}$ à chaque fois la même erreur de lecture ${ }^{17}$, et qu'il est tout à fait légitime, quand la fausse autorité du reputari de $\mathrm{Z}$ a été ruinée par le praesentari de $\mathrm{A}$, de supposer que $\mathrm{A}$ portait praesentabo là où nous lisons dans $\mathrm{Z}$ reputabo.

Pontieri, qui ne répond pas aux exigences d'une édition savante, a imprimé reputari sans faire état de sa variante dans l'apparat, ne permettant pas à la critique de vérifier son choix ${ }^{18}$.

risquant à prendre à la lettre reputari, avance une interprétation inadmissible ( I I am asking that this work be considered to have come from you or at least to have been written under your aegis»).

16. Comparer, à titre d'exemple, Orderic Vital, prol. in aeccl. hystoriam (p. 132,5-9 Chibnall [= I 3-4 Le Prevost]) [...] Praecipuam nempe in hoc fiduciam habeo, quod hoc opus incepi uenerandi senis Rogerii abbatis simplici precepto tibique, pater Guarine, qui secundum aecclesiae ritum ei legitime succedis, exhibeo, ut superflua delens incomposita corrigas et emendata uestrae sagacitatis auctoritate munias - perpétuant d'ailleurs un usage antique: cf. Pline, epist. 1, 2, 1 [...] librum quem prioribus epistulis promiseram [...] rogo ex consuetudine tua et legas et emendes; 7, 20, 1-2 (à Tacite) Librum tuum [il s'agit ou du Dialogue sur les orateurs ou, plus sûrement, d'un livre des Histoires] legi et quam diligentissime potui adnotaui quae commutanda, quae eximenda arbitrarer. [...] Nunc a te librum meum cum adnotationibus tuis exspecto; Justin, praef. 5, Quod ad te non cognoscendi magis quam emendandi causa transmisi. Voir Simon 1959-1960, 124-130 - citant Malaterra, n. 126, dans la version de Pontieri.

17. La faute est aisément explicable: pnt- + tilde (abréviation par contraction de praesent-) a été transcrit put-, suite à un déchiffrement erroné des jambages et du signe abréviatif.

18. Si l'on ne tient pas compte des changements de graphie et des bévues manifestes de A, on relève dix-neuf autres endroits au moins de la première épître dédicatoire pour lesquels l'éditeur italien ne mentionne aucune des lectures divergentes de A. Voici la liste de ces données que Pontieri ne met pas à la disposition de la critique: nostrae] beatae A (p. 3,2); Cathanensium] cathaniensium A (p. 3,2); infelici] felici A (p. 3,3); Quandoquidem] quoniam quidem A (p. 3,5); quo concingimur] et contimur A (p. 3,7); aut (saltem)] ad (saltim) A (p. 3,9); ob reuerentiam] reuerentia A (p. 3,10 sq.); accesserit] accesserius A (p. 3,12); si] om. A (p. 3,12); haec... adscribantur] hoc... ascribatis A (p. 3,14); tam... quam] iam... sed A (p. 3,14); de] me A (p. 3,14); interfuissem] interfuisse A (p. 3,15); nouiter] om. A (p. 3,16); limpidius] limpidus A (p. 3,17); potuissem] potuisse A (p. 3,18); patesceret] patescere A (p. 3,19); innixus] municus (pour munitus [les quatre premiers signes forment une suite de huit jambages indistinctement différenciés, qu'il n'est guère aisé d'interpréter]) A (p. 3,21); nostro] uestro $\mathrm{A}(\mathrm{p} .3,22)$. Ainsi douze variantes authentiques de $\mathrm{A}$ sont envoyées à la trappe. Aux vingt omissions de cet apparat insincère (cf. Resta 1964, 24) s'ajoutent deux fausses lectures: fratri (p. 3,4 [app.] pour fratre); eruchiare (p. 3,18 [app.] pour eructriare [sic]), et une mention fallacieuse: factum om. A (p 3,16 [app.]), lequel porte en réalité factrum (sic). Avec l'édition princeps (P), Pontieri n'a pas usé de plus de ménagements: «Un attento vaglio dell'apparato rivela che parecchie lezioni sono arbitrariamente attribuite a $P$, mentre molte lezioni di $P$ non sono registrate $[\ldots]$ (.. (Resta 1964, 16). Nous signalerons seulement que, p. 3,21-22 (app.), la dernière unité critique porte au compte de $\mathrm{P}$ deux séries de lectures contradictoires: nostro principi / Principis nostri et sustentamine gratiosior fruar/sustentamine fruar - doublons passablement déconcertants pour tout lecteur soucieux de reconstituer à partir des variantes consignées dans l'apparat les différents états du texte. 
Nous nous sommes arrêté à une troisième phrase de la vulgate imprimée, dont l'allure boiteuse s'explique très probablement par une lecture incorrecte du texte originel. Dans (p. 4,32-36) alii [...] gressum mentis semper in humilitate figentes, aliorum dicta uel facta non remordentes, sed quod minus ornate dictum ab eis audierunt cum mansuetudine intra seipsos, non autem in publico, ne forte scandalizentur, corrigunt, la particule adversative sed crée un déséquilibre formel manifeste en opposant à deux participes présents juxtaposés, figentes et remordentes, une forme verbale personnelle, corrigunt. Or, le premier des deux participes présents (figentes ${ }^{19}$ ) définit une attitude d'âme, une disposition intérieure constantes dont les actions désignées ensuite, que ce soit par le second participe (remordentes) ou par la forme verbale personnelle (corrigunt), sont des manifestations objectivement observées. Dès lors, puisque le second participe présent se révèle à l'analyse être le prolongement du premier au même titre que le segment de phrase coordonné par sed (sed... corrigunt $)^{20}$, il parait difficile de ne pas aligner remordentes sur corrigunt et ne pas donner à imprimer remordent ${ }^{21}$ - à moins de considérer que ce participe présent fait fonction de verbum finitum dans une proposition indépendante ${ }^{22}$, ce qui, à notre connaissance, serait le seul endroit de la chronique malaterranéenne où cette construction se rencontre ${ }^{23}$.

\section{II}

Même débarrassé des fantaisies que l'édition princeps et Pontieri y ont introduites, le texte de Malaterra renferme encore plus d'un passage qu'on ne saurait regarder comme établi d'une manière définitive. Il s'agit de phrases dont la construction et la

19. L'expression gressum mentis [...] in humilitate figere reparaît en IV 15 (p. 94,4 sq.), sous une forme partiellement renouvelée, pour caractériser les dispositions de Roger au terme de la conquête de la Sicile: [...] studio agebat ut in perfectae humilitatis statu persistens gressum mentis figat.

20. On peut appliquer ici à sed ce que Gardes-Tamine \& Pellizza 1998, 28, disent de «mais»: «Le sens de cette conjonction adversative suppose que l'on ait présent en tête ce à quoi va s'opposer la proposition qu'elle introduit. Elle lie donc doublement les propositions qu'elle rattache». Il existe une égalité fonctionnelle entre le second syntagme participial (remordentes) et la proposition qui lui est «enchaînée» par sed. En revanche, les deux participes ne sont pas sur le même plan énonciatif, le second, nous l'avons vu, offrant une première illustration du participe antécédent. Sur la traduction de sed, voir Krekelberg \& Remy 1967, 32, \$ 44. Remarques, 2.

21. Non seulement la phrase devient ainsi plus symétrique, mais la première des propositions coordonnées reçoit avec cette correction une forme syntaxique qui en fait le pendant exact du passage de la même lettre dénonçant l'attitude contraire: (p. 4,30) opus alienum detrahendo mordaci dente appetere non desistunt.

22. Voir Liberman 2010, 396 (ad v. 92).

23. Sur un participe passé faisant fonction de verbum finitum dans une proposition subordonnée, voir Desbordes 2005, 148 (ad II 37 [p. 47,19-21]). 
grammaire ne semblent pas en défaut, mais auxquelles il ne parait guère possible de donner une interprétation satisfaisante. Or, nous avons remarqué que l'hypothèse d'une mutilation peut, dans certains de ces endroits, faire avancer la critique et l'intelligence du texte.

I 27 (p. 21,17-20) Pecunias habentes, quod emerent non habentes atque ipsos liberos ex ingenuitate procreatos uili pretio in seruitio uenundantes, dum ubi illud ad uictus utilitatem expenderetur non inueniebant, ad augmentum doloris sui amissione incassa uenditione liberorum quasi quarta calamitate cruciabantur.

quod $\mathrm{C}$ : quid $\mathrm{ZB}$, edd. || atque edd.: at $\mathrm{Z}$ autem $\mathrm{C}$ om. B || ipsos $\mathrm{C} \mathrm{Z}$ : suos $\mathrm{B}$ || procreatos $\mathrm{C} \mathrm{Z}$ : plorantes $\mathrm{B}$, Pontieri || seruitio $\mathrm{C}$ : -tium $\mathrm{ZB}$, edd. || ubi C B: ibi Z || expenderetur C: -rentur Z -rent B || amissione ed. pr.: amissionem C Z propter amissionem B, Pontieri.

Considérée en elle-même, l'ouverture de cette phrase, embrouillée jusqu'au paradoxe, soulève de grosses difficultés: pourquoi des parents fortunés en seraient-ils réduits, dans ce temps de disette, à vendre leurs enfants "à vil prix", alors même qu'avant de s'y résoudre, ils ne trouvaient pas de denrées à acheter? Et pourquoi, après ce parti extrême, s'opiniâtrent-ils dans leur quête de nourriture, mais en ne consacrant à l'acquisition de vivres que le produit de cette vente ${ }^{24}$ ? Il y a dans un tel comportement plusieurs défis au bon sens.

Si nous examinons les variantes, nous observons qu'une des leçons que fait connaître $\mathrm{C}$, autem, a cédé la place à une correction indiquée par l'éditeur princeps, $a^{2} q u e^{25}$. Or, si l'on rétablit autem, on donne à cette phrase une forme plus riche de sens. En effet, appelant une autre distribution des termes que celle que la vulgate a imposée, ce rétablissement conduit à une nouvelle liaison d'idées ${ }^{26}$ - à pecunias habentes s'oppose <pecunias > non habentes -, puis à constater l'existence d'une perte de texte après emerent: Pecunias habentes, quod emerent $\langle\ldots\rangle$; non habentes autem, ipsos liberos [...] uenundantes, eqs. Telle qu'elle a été transmise, cette phrase est incomplète: elle ne dit pas comment les riches se sont comportés face à cette situation de pénurie. En revanche, elle évoque les déchirements qu'ont éprouvés les pauvres, ceux qui n'avaient que leur progéniture pour richesse: ayant pensé se procurer des moyens de subsistance en vendant leurs enfants et constaté l'inutilité de leur sacrifice, vu qu'il n'y avait rien, nulle part, à acheter, ils étaient la proie de remords torturants.

24. En effet, dans dum ubi illud ad uictus utilitatem expenderetur non inueniebant, le pronom neutre illud ne peut être référé qu'à uili pretio.

25. Selon toute apparence, atque provient du at de Z, qui, d'ailleurs, assorti d'un signe abréviatif, représente $a(u) t(\mathrm{em})$ plutôt que at (que).

26. Autem introduit un parallèle entre deux membres de phrase tenus jusqu'ici pour étroitement liés sur le plan de la syntaxe et entraîne un changement dans la ponctuation. 
II 35 (p. 45,31-33) Vnde [sc. e prouincia Agrigentina] cum maxima cum praeda rediret, partem militum suorum praedam praecedere praecipiens, ipse cum reliquis inter duos cuneos minatur, ne a fronte uel certe a tergo aliquo hostili incursu excuteretur. praedam $\mathrm{ZB}$ : praeda $\mathrm{C}$.

Cette phrase est obtenue en prenant pour fondement de la constitution du texte le ms. C, rectifié sur un point. De son côté, Pontieri a établi de nouveau ici un texte composite, amalgamant les données que les différents états de la tradition lui présentaient. Se soustrayant en apparence à l'influence de $\mathrm{P}^{27}$ (Cum maxima praeda inde rediret), qu'il relègue dans l'apparat, il privilégie l'accord C B sur Vnde cum; puis il abandonne $\mathrm{C}$ pour adopter, en le retouchant, le dessin syntaxique redéfini par $\mathrm{B}$ pour remédier à la chute de praeda: rediret, cum maxima parte militum suorum praedam praecedere praecipiens. Enfin, devant les incertitudes de cette source ${ }^{28}$, il revient à $\mathrm{C}$ et $\mathrm{P}$, dont les témoignages concordent exactement: ipse cum reliquis inter duos cuneos minatur, ne a fronte uel certe a tergo aliquo hostili incursu excuteretur. Reste que, sous quelque forme qu'on l'examine, cette phrase souffre plus d'une incohérence. Ainsi, à titre d'exemple - et sans vouloir préjuger les questions soulevées par minatur -, comment Roger pourrait-il se retrouver, avec les hommes qui restent à sa disposition, inter duos cuneos, après avoir seulement détaché en avant une partie de sa troupe?

Si l'on considère les autres indices matériels fournis par Malaterra sur cette péripétie de la conquête, il est clair que la petite colonne formée par Roger et ses deux cents chevaliers a adopté la disposition habituelle de l'agmen romain progressant en pays ennemi: une pars militum ouvre la marche ${ }^{29}$, pour parer à toute attaque frontale; suivent les écuyers conduisant le train des chariots ou les bêtes de somme qui transportent le butin ${ }^{30}$; et Roger lui-même, cum reliquis, ferme la marche à peu de distance, pour déjouer toute entreprise hostile par l'arrière ${ }^{31}$. L'expression inter duos cuneos (p. 45,32) s'interprète dans ces circonstances d'une manière naturelle: le butin occupe le centre de la colonne; il est précédé et suivi d'un cuneus de chevaliers.

27. Contrairement à ce que Pontieri indique dans son apparat, $\mathrm{P}$ a reproduit le doublon qu'il lisait dans $\mathrm{Z}$ (Vnde... inde). Ce inde serait-il le développement aberrant d'un cum réduit aux deux premiers signes surmontés d'un tilde?

28. De toute évidence, $\mathrm{B}$ ne lisait pas dans son modèle le minatur que $\mathrm{C}$ et $\mathrm{Z}$ ont transcrit sans aucune équivoque: il a commencé par tracer quatre "jambages» anormalement espacés, qui n'offrent aucune apparence de sens; la suite, en revanche, se déchiffre aisément (-itatur) - sans pour autant rendre intelligible le mot entier, et il est impossible d'y reconnaître, avec Pontieri, inuitatur. D'autre part, certe a est tombé devant tergo.

29. Cf., outre partem militum suorum praedam praecedere praecipiens (p. 45,31 sq.), nostri [...] qui praecedebant (p. 46,2).

30. Cf. parte armigerum (sic) qui praedam minabant perempta (p. 46,6).

31. Cf. Comes [...] qui subsequebatur (p. 46,7). 
Au moment de l'attaque surprise des ennemis, l'avant-garde s'est débandée sur-lechamp $^{32}$; les écuyers sont en partie massacrés et le butin que les Normands avaient amassé est pillé ${ }^{33}$; Roger remonte alors de l'arrière pour tenter de rallier sa troupe en désordre ${ }^{34}$.

L'examen des faits démontre donc à l'évidence que, dans le morceau de texte commençant avec inter duos cuneos, "ce dont il est question", "ce dont parle le reste de la phrase» renvoie au butin, à la praeda, que les verbes minatur et excuteretur ${ }^{35}$ ne peuvent avoir ipse cum reliquis - c'est-à-dire Roger - pour sujet, qu'il faut marquer une lacune après reliquis. La phrase originelle a été amputée accidentellement d'une partie de sa substance: [...] ipse cum reliquis $<\ldots$.. praeda ${ }^{36}>$ inter duos cuneos minatur, ne a fronte uel certe a tergo aliquo hostili incursu excuteretur.

32. Cf. Nostri $[\ldots]$ qui praecedebant [...], illis ex improuiso insurgentibus, $[\ldots]$ cum fuga potius quam armis mortis periculum declinare cupiunt (p. 46,2-4).

33. Cf. Hostes [...], parte armigerum (sic) qui praedam minabant perempta, ipsam etiam praedam excutiunt (p. 46,6 sq.).

34. Cf. Comes [...], tumultum audiens, [...] citius aduolat [...] (p. 46,7 sq.).

35. Comme excuteretur, minatur ici est un passif; cf. Flobert 1975, 306; 378. Minare se dit des bêtes et des gens qu'on «mène» ou «emmène », d'un chariot que l'on «conduit»: cf., par exemple - et pour se limiter à la Vulgate -, Ex 3, 1 cumque minasset gregem ad interiora deserti; 1 S 30, 20 et tulit uniuersos greges et armenta et minauit ante faciem suam; 1 Par 13, 7 Oza autem et frater eius minabant plaustrum; Is 11, 6 uitulus et leo et ouis simul morabuntur et puer paruulus minabit eos; Act 18, 16 et [Gallio, proconsul Achaiae,] minauit eos [sc. Paulum et Iudaeos] a tribunali; etc. Malaterra ne l'emploie guère qu'avec praeda: cf. I 16 (p. 17,6) paucis qui praedam post se minent (A Z: ducant B, Pontieri) relictis; III 11 (p. 63,28 sq.) [praedam] nauibus introducendam illorsum minare accelerat. Les interprètes modernes de Pontieri, qui ont dépensé des trésors d'imagination pour se représenter et exposer l'ensemble de la situation avec autant de précision que le latin de cette édition le permet, font tous bon marché de ce terme. Quant à excutere, nous ne croyons pas nous tromper en affirmant que, dans la chronique, les formes passives de ce verbe ne se rencontrent que dans les cas de transformation passive du tour praedam excutere (trois exemples de la construction active - cf. I 16 [p. 16,34]; II 35 [p. 46,6, cité supra] et III 11 [p. 63,31] - contre deux de son homologue passive, toutes deux en II 35 [p. 45,33 et 46,19 sq., praeda excussa recuperatur]). Bien qu'elle soit attestée en latin classique (cf. Columelle, 7, 12, 9 [canis pecuarius] ad rixam pugnamque nec tantum ad cursum comparatur, cum et lupi repellere insidias et raptorem ferum consequi fugientem praedamque excutere atque auferre debeat; et Ammien Marcellin, hist. 27, 8, 7 praedam excussit; 30, 4, 21 excutiunt praedas; 30, 7, 8 praeda raptoribus ui fractis excussa), cette expression provient plus sûrement de la Bible (cf. $1 \mathrm{~S}$ 30, 8 absque dubio enim conprehendes eos [sc. latrunculos] et excuties praedam). Elle se retrouve chez Grégoire de Tours, hist. 10, 5 (p. 336,7 Buchner), et Paul Diacre, hist. 3, 9 (p. 194,8 Schwarz); 4, 39 (p. 248,36 Schwarz), mais aussi dans des annales: cf. Ann. Bertiniani a. 882 (MGH I, p. 515 Pertz) maxime autem ex illis [sc. Nortmannis] qui Remum adierunt [...] praedam excussit, et Ann. Fuldenses a. 883 (MGH I, p. $398^{\mathrm{A}}$ Pertz) [...] non paucos ex eis [sc. Nordmannis] prostrauit et praedam excussit.

36. La restitution ne fait pas de doute. Mais il est, bien évidemment, difficile de retrouver ce que Malaterra avait écrit. Tout au plus peut-on conjecturer qu'il avait exprimé l'idée que le comte Roger, avec ce qu'il lui restait de chevaliers, constituait, derrière le butin, l'arrière-garde de la colonne: ipse cum reliquis <subsequitur $>$. Et il n'est pas exclu qu'après la chute du verbe dont ipse est le sujet et celle 
Autre passage de la chronique dont l'altération présumée nous parait résulter d'une lacune:

II 40 (p. 49,14 sq.) quae usui capiendae urbis necessaria erant [...]

usui $\mathrm{ZB}$ : usum $\mathrm{C}$

Bien que la construction dative du complément de necessarius se rencontre occasionnellement dans la chronique ${ }^{37}$, la lecture des manuscrits $\mathrm{Z}$ et $\mathrm{B}$, usui, qui a passé dans toutes les éditions, ne mérite pas une entière créance. En effet, on ne comprend pas pourquoi, si cette leçon était le texte original de Malaterra, le datif se serait altéré dans $\mathrm{C}$ en un accusatif. En revanche, on s'explique aisément le changement contraire: l'accusatif seul ne pouvant être conservé sans modification, pour faire disparaître ce que la construction héritée de l'hyparchétype a d'illogique, un ancêtre de ZB aura changé usum en usui. Il n'y a donc rien de téméraire à récuser le témoignage de ces derniers : telle quelle, la variante de $\mathrm{C}$ est évidemment mauvaise; mais au rebours d'usui, elle est "authentique » ${ }^{38}$, et elle donne à penser qu'ici, comme en bien d'autres endroits ${ }^{39}$, Malaterra avait écrit ad usum ${ }^{40}$.

Olivier DesBordes

Centre Michel de Boüard - CRAHAM (UMR 6273)

Université de Caen Basse-Normandie

du nom praeda, sujet de minari, ce dernier mot, mis en relation avec ipse, n'ait été arrangé pour restaurer la régularité grammaticale de la construction provenue de l'amputation de la rédaction primitive: ipse cum reliquis < subsequitur, ita ut praeda > inter duos cuneos minaretur, ne a fronte uel certe a tergo aliquo hostili incursu excuteretur. Cuneus, dont on ne peut citer qu'un autre exemple dans la chronique (cf. II 33 [p. 43,30] duos cuneos faciens ex suis), est utilisé sans propriété, dans un sens très étendu, éloigné de son acception militaire traditionnelle. Cet usage correspond aux emplois que la Vulgate fait de ce terme: cf. Nb 1, 52; Dt 20, 9; Jos 4, 13; Jg 9, 37; 9, 43-44; 1 S 13, 17 (bis); 30, 15; 2 S 2, 25.

37. Cf., par exemple, III 13 (p. 65,14) [...] donec ea quae tanto inceptui necessaria forent apparabantur; IV 17 (p. 96,14 sq.) arma et quae defensioni necessaria erant aptant.

38. Une leçon est «authentique», «quand elle a été conservée par une tradition ininterrompue» (Havet $1911,425, \$ 1623)$.

39. Cf. I 10 (p. 13,19) cetera quae ad usum necessaria erant; ibid. (p. 13,20 sq.) [...] machinamentis [...] ad id officii agendum necessariis aptatis; I $18(\mathrm{p} .18,19)$ his quae ad expeditionem necessaria erant $<$ apparatis> ; I 20 (p. 19,16) omnibus quae ad uictum necessaria erant [...] introductis; I 21 (p. 19,23 sq.) paratis his quae ad expeditionem necessaria erant; I 21 (p. 19,26) omnia [...] quae ad uictum necessaria erant; II 30 (p. 41,24 sq.) quae ad usum necessaria erant; IV 4 (p. 87,18 sq.) sumptus ad id negotii necessarii; IV 23 (p. 101,19) sumptibus ad id quod coeperat necessariis. Comparer, par exemple, Tite-Live 2, 32, 4 rem nullam nisi necessariam ad uictum sumendo, ou Orderic Vital, hist. eccl. 10,6 (p. 222, 10 Chibnall = IV 29 Le Prevost) [Insulam Man] domibus et aliis necessariis ad usus hominum gnauiter instruxit.

40. On sait que l'omission des mots courts est habituelle et de toutes les époques, qu'il est vain, par conséquent, d'en chercher l'explication. Mais il reste qu'en I 3 (p. 9,1 sq.), le classique « saut du même au même» aura peut-être provoqué la chute de la préposition ad devant altioris: [...] heredum Dei 


\section{Références bibliographiques}

D’Angelo E. (2003), Storiografi e cronologi latini del Mezzogiorno normanno-svevo, Naples, Liguori Editore (Nuovo Medioevo; 69).

Desbordes O. (2005), «Nouvelles notes critiques sur les deux premiers livres de la chronique de Geoffroi Malaterra», Kentron, 21, p. 111-159.

Diggle J., Goodyear F.R.D. (1972), The Classical Papers of A.E. Housman, Cambridge, Cambridge University Press, 3 vol.

Flobert P. (1975), Les verbes déponents latins des origines à Charlemagne, Paris, Les Belles Lettres (Publications de la Sorbonne. Série «NS Recherches»; 17).

Gardes-Tamine J., Pellizza M.-A. (1998), La construction du texte. De la grammaire au style, Paris, Armand Colin (Cursus, série «Lettres»).

Havet L. (1911), Manuel de critique verbale appliquée aux textes latins, Paris, Librairie Hachette \& $C^{\text {ie }}$ (réimpr. anastatique: Rome, «L'Erma» di Bretschneider, 1967).

Krebs J.P., Schmalz J.H. (1905), Antibarbarus der lateinischen Sprache, $7^{e}$ éd., Bâle - Stuttgart, Benno Schwabe \& Co. AG (réimpr. Darmstadt, Wissenschaftliche Buchgesellschaft, 1984), I-II Bd.

Krekelberg J., Remy E. (1967), Les formes typiques de liaison et d'argumentation dans l'éloquence latine, entièrement revu et corrigé par A. Maniet, Namur, WesmaelCharlier.

Liberman G. (2010), Stace, Silves. Édition et commentaire critiques, Paris, Éditions Calepinus (Littérature latine).

Lo Curto V. (2002), Goffredo Malaterra, Ruggero I e Roberto il Guiscardo, Cassino, Francesco Ciolfi Editore.

Loud G.A. (2005), Geoffrey Malaterra, The Deeds of Count Roger of Calabria \& Sicily \& of Duke Robert Guiscard his brother (unpublished translation), 86 p. (fichier PDF).

adiutorio et sua strenuitate gradatim $<a d>$ altioris honoris culmen scandentium. Ici, la préposition ne s'impose pas par un caractère de nécessité absolue: le complément du verbe scandere est tantôt relié directement au verbe, tantôt introduit par une préposition; cf. Krebs \& Schmalz 1905, II, 538. Cependant, I 23 (p. 20,7 sq.) - seul autre endroit de la chronique où ce verbe est employé - rend ce supplément très vraisemblable: haud [...] indignum quin gradatim ad alta, ut ille [sc. Robert Guiscard] fecerat, etiam ipse [sc. Roger], fortuna sibi fauente, scanderet. Comparer Dhuoda, lib. manualis 1, 1: Inter quos adortor te, fili, ut, in quantum uales, illa semper perquiras ubi cum dignis et abtis deumque diligentibus ad certum possis scandere culmen [...]. On nous permettra de faire observer, en passant, qu'on ne peut suivre Pontieri quand il imprime ici, d'après $\mathrm{C}$, et iam ipse. Notons, en outre, qu'en II 14 (p.33,17 sq.) cum maxima laetitia recedentes et, debilitate gentis cognita, audaciores, le choix de la leçon introduite dans le texte par l'édition princeps, et, ne doit pas être ratifié. Il n'y a pas lieu néanmoins de lui substituer la variante des mss, etiam, que l'éditeur italien a négligé de faire connaitre: pour retrouver la vraie leçon, il faut décomposer la forme transmise, née de la coalescence de la coordination et et de l'adverbe iam. 
Lucas-Avenel M.-A. (2001), Édition critique avec traduction et commentaire des deux premiers livres de la chronique de Geoffroi Malaterra, thèse de doctorat de langues et littératures anciennes sous la direction de L. Callebat et S. Fodale, Université de Caen Basse-Normandie, 4 fasc. (I : 1-148 p.; II : 149-29o p.; III : 291-430 p.; IV : 431603 p.; I-VI: résumé italien; 9 hors-texte; table des matières) (dactyl.).

LucAs-Avenel M.-A. (2008), «La nouvelle édition de la chronique de Geoffroi Malaterra », ArNo-S, I, p. 31-49.

Muratori L.A. (1724), Gaufredi Malaterrae monachi Benedictini historia Sicula ad fidem veterum manuscriptorum a Joanne Baptista Carusio edita, nunc iterum cum prioribus editionibus collata, multisque mendis expurgata, in Rerum Italicarum scriptores ab anno aerae Christianae quingentesimo ad millesimum quingentesimum [...], Tomus quintus, Milan, Ex typographia societatis Palatinae in Regia curia, p. 537-602.

Pontieri E. (1927-1928), De rebus gestis Rogerii Calabriae et Siciliae Comitis et Roberti Guiscardi Ducis fratris eius auctore Gaufredo Malaterra monacho Benedictino, Bologne, Nicola Zanichelli (Rerum Italicarum Scriptores. Raccolta degli Storici italiani dal cinquecento al millecinquecento ordinata da L.A. Muratori. Nuova edizione riveduta ampliata e corretta con la direzione di G. Carducci - V. Fiorini P. Fedele. Tomo V - Parte I; Fasc. 1; 2-3).

Resta G. (1964), «Per il testo di Malaterra e di altre cronache meridionali », in Studi per il CL anno del Liceo-Ginnasio «T. Campanella» (1814-1964), Reggio Calabria, p. 3-6o.

SimON G. (1958; 1959-1960), «Untersuchungen zur Topik der Widmungsbriefe mittelalterlicher Geschichtsschreiber bis zum Ende des 12. Jahrhunderts », Archiv für Diplomatik, Schriftgeschichte, Siegel- und Wappenkunde, 4, p. 52-119 (Erster Teil); 5/6, p. 73-153 (Zweiter Teil).

Toubert P. (2005), «L'historiographie italo-normande au XI ${ }^{\mathrm{e}}$ siècle. À propos d'une édition récente», Le Moyen Âge, t. CXI, 2, p. 351-354.

Wolf K.B. (2005), Geoffrey Malaterra, The Deeds of Count Roger of Calabria and Sicily and of his Brother Duke Robert Guiscard, Ann Arbor, The University of Michigan Press. 